\title{
TADEEC: Threshold Sensitive Advanced Distributed Energy Efficient Clustering Routing Protocol for Wireless Sensor Networks
}

\author{
Anamika Chauhan \\ Department Of IT \\ Delhi Technological University \\ New Delhi, India
}

\author{
Amit Kaushik \\ Department Of IT \\ Delhi Technological University \\ New Delhi, India
}

\begin{abstract}
In Wireless sensor networks, several routing protocols have been developed to improve the various parameters such as stability period, throughput etc .After a homogenous protocol leach, many heterogeneous protocol were developed which successfully improved the routing in WSNs .In this Paper we studied one of the heterogeneous protocol DEEC and its improved version EDEEC and we tried to work on two parameters i.e. Stability period and throughput of the network to improve these further .In this Paper we introduced a new node i.e. super advanced node with the existing heterogeneous nodes in the EDEEC which successfully improved its stability period and we also incorporated a reactive protocol i.e. TEEN in our paper to make our network communication more efficient .Thus we used the best of EDEEC and TEEN and made TADEEC protocol. This has been simulated in Matlab and results have outperformed the LEACH, DEEC, EDEEC etc.
\end{abstract}

\section{Keywords: EDEEC, DEEC, TEEN}

\section{INTRODUCTION}

Wireless Sensor Network (WSN) is a kind of self-configuring network composed of a set of wireless sensor nodes also called motes. The lifetime of WSN is limited because the sensor node works on battery life and it is hard to recharge the battery at regular basis because of the deployment of wireless sensor network in remote and hostile places. The energy cost is more in term of transmission of data than processing data. In order to prolong the network lifetime, a network routing protocol with high energy efficiency is necessary besides designing low-power sensor nodes. A current research challenge is to develop low-power communication with lowcost on-node processing and self-organized connectivity/protocols. Several protocols were developed to make the communication energy-effective to prolong the life of the networks. These protocols were different in how they improve the communication and transmission of the packets in the network but they all based on clustering approach in the network. The homogeneous protocol(due to same initial energies of all nodes) LEACH[1] which considered all nodes of same energy used the probabilistic approach to elect the cluster head and the probability of choosing the cluster head decides after how many rounds a node can be again cluster head. But this approach does not guarantee for a high energy node to be cluster head. PEGASIS [3] was proposed to do cluster-free routing. In this sensor nodes form a chain, they transmits and receives data from a neighbor, neighbor node is selected on the basis of energy status and only one from that chain transmit data to the base station. In this a sensor node should know the energy status of its neighbors in order to route its data, such topology adjustment can introduce significant overhead for highly utilized networks. After these homogenous, a heterogeneous protocol came, SEP[4] which divided the network into two types of nodes having different energy normal and advanced nodes. The probabilistic method of selecting the cluster heads is different for these two types of nodes. These probabilities are based on their energies. This was done to improve the stability period (the time when first node dies [4]) of the network and to extend the life. Several enhanced versions of SEP routing schemes were proposed such as ESEP, ZSEP etc. ESEP [5] increase the heterogeneity to level 3 using the normal, intermediate and advanced nodes and these were taken in the order of increasing initial energies resp. ZSEP[6] divided the network in the form of zones which resemble clusters so that proper distribution of the nodes and energies in each cluster can be done These successfully outperformed the SEP but shortcoming of these protocols including SEP of not considering the effect of residual energies on the cluster head selection probabilities for different kind of nodes. Another Protocol, DEEC [7] took the concept of residual energy and average energy of the network in electing the cluster heads with existing heterogeneity of the nodes and successfully improved the routing in network. Several versions of DEEC were explored which improved it further such as DDEEC and EDEEC. DDEEC [8] uses same method for estimation of average energy in the network and $\mathrm{CH}$ selection algorithm based on residual energy as used in DEEC. DDEEC introduces threshold residual energy and when energy level of advanced and normal nodes falls down to the limit of threshold residual energy then both type(normal and advanced) of nodes use same probability to become cluster head. EDEEC [9], which was enhanced version of DEEC proposed to insert another node in the network (super node) with the existing normal and advanced nodes which increased the heterogeneity and lifetime as well. It has been evaluated in [10] that DDEEC has low stability period, lifetime and throughput as compared to the EDEEC. So EDEEC act as motivating factor to work on and improve it further. TEEN[12] which was a threshold sensitive reactive protocol proposed scheme to minimize the transmission time as transmission consume more energy than processing of data at the nodes. This was done to impart two threshold parameters, hard and soft threshold. A node only transmit when currently sensed value is greater than hard threshold and difference between current sensed and previous sensed value is greater than soft threshold .This protocol is a kind of 
optimization technique which can optimize the communication in a good manner.

In this paper we worked on EDEEC and TEEN, and successfully approached to make use of the best of these protocols and our protocol TADEEC successfully improved the stability period and throughput of the network which ultimately enhanced the life of the network. This paper is divided as II section is about related research section III is about the proposed protocol TADEEC and section IV and V about simulation and results and conclusion resp.

\section{RESEARCH BACKGROUND}

SEP introduced level two heterogeneity with normal and advanced nodes with advanced nodes have ' $a$ ' times more energy than normal where 'a' can have value between 0 and 1.It maintained a concept of subepochs of advanced nodes within a epoch of a normal node. An epoch is number of rounds after a node again become eligible to become a cluster head. In SEP the heterogeneity imparts subepochs in the epoch of normal nodes and the advanced nodes are eligible for cluster head in these subepochs. Thus SEP increase lifetime of normal nodes by electing more energy nodes (advanced) to become cluster head rapidly than normal nodes. The cluster head probability as in leach is $p_{\text {opt }}$ so no of cluster heads in a round is $n * p_{\text {opt }}$. DEEC used the ratio of residual energy left after communication $\left(E_{i}(r)\right)$ and average energy $(E(\bar{r}))$ of the nodes in election of cluster heads as

$$
\begin{aligned}
& Y=E_{i}(r) / E(\bar{r}) \\
& E(\bar{r})=\frac{1}{n} * E_{\text {total }}(1-(r / R))
\end{aligned}
$$

$\mathrm{n}$ is total no of nodes is total no of rounds (lifetime) and $\mathrm{r}$ is current round no. $\mathrm{E}_{\text {total }}$ is the total energy of the nodes. In EDEEC , the heterogeneity level is increased and three types of nodes normal, advanced and super are in the network. The energy of advanced and super nodes are ' $a$ ' and ' $b$ ' times than normal nodes resp.EDEEC maintain no of cluster heads as $n * p_{\text {opt }}$ in a round Thus

$$
p_{\text {norm }} * n_{\text {norm }}+p_{a d v} * n_{a d v}+p_{\text {sup }} * n_{\text {sup }}=n * p_{\text {opt }}
$$

Introducing the third node type increased the epoch times for normal nodes to become cluster heads and thus cluster heads are made in this way such that more energy nodes become cluster heads earlier than lower energy nodes. Thus increasing the longevity of less energy nodes. It is wastage of time and energy for cluster heads of network to send similar type of data to base station again and again. This problem is eliminated by TEEN using thresholding approach. Being a reactive protocol it act reactively by checking the data significance and decide to transmit or not. TEEN implement this approach using two thresholds hard and soft threshold.

\section{PROPOSED APPROACH}

Our approach is minimize transmission time in the network. The basic theory is that clustering is done because the nodes which are clustered have a sensed data which vary in very insignificant amount. So cluster head in a cluster when take the data from their members is similar in nature. Cluster heads have to send similar type of data again and again to base station which is time consuming and wastage of energy by the cluster heads. This concept was explored in detail in TEEN which imposed two thresholds hard and soft threshold which optimized the communication and prolong the life of the network. We tried to use the optimization Protocol TEEN on enhanced version of DEEC i.e. EDEEC. But we according to our scheme we first increased the stability period by introducing a new node "superadvanced" in our network. Introducing a node increased the heterogeneity to level four but thing is that it is not using nodes having energy more than super nodes as in EDEEC. So nodes are in our scheme are

normal nodes: $E_{0} \quad$ advanced: $E_{0}(1+a)$ super: $E_{0}(1+b) \quad$ superadvanced: $E_{0}(1+c)$ where $a=c / 2 ; b=3 c / 4 ; c=1$;

Here $p_{\text {opt }}$ is probability of choosing the cluster heads in the network so a node become eligible for cluster head again after $1 / p_{0}$ rounds. So average no of cluster heads should be $n * p_{\text {opt }}$ if $\mathrm{n}$ is total no of nodes. In our scheme nodes are distributed according to constant $\mathrm{m}$ and $\mathrm{m} 0$ and nodes are:

normal $=(1-m) * n$;

advanced $=(1-m 0) * m * n$;

super $=(m 0 * m * n) / 2$;

supadvanced $=(m 0 * m * n) / 2$;

total energy of the network in a round is

$E_{0} *(1-m) * n+E_{0}(1+a) *(1-m 0) * m * n$

$+E_{0}(1+b) *(m 0 * m * n) / 2+E_{0}(1+c) *(m 0 * m$

$* n) / 2=n * E_{0}(1+a * m-m * m 0 *(a-((b+c) / 2))$;

So, weighed probabilities of different nodes are

$p_{\text {norm }}=\frac{p_{\text {opt }}}{(1+a * m-m * m 0 *(a-((b+c) / 2))}$

$$
p_{a d v}=\frac{p_{o p t} *(1+a)}{(1+a * m-m * m 0 *(a-((b+c) / 2))}
$$

$p_{\text {super }}=\frac{p_{o p t} *(1+b)}{(1+a * m-m * m 0 *(a-((b+c) / 2))}$

$$
p_{s a d v}=\frac{p_{o p t} *(1+c)}{(1+a * m-m * m 0 *(a-((b+c) / 2))}
$$

Also,

$p_{\text {norm }} *(1-m) * n+p_{a d v} *(1-m 0) * m * n+$

$+p_{\text {sup }} *(m 0 * m * n) / 2+p_{\text {sadv }} *(m 0 * m * n) / 2$

$=n * p_{\text {opt }}$

It clearly shows the correctness of this proposed protocol as it is specified in SEP that for proper distribution of energy, this condition of total no of cluster heads equal to $n * p_{\text {opt }}$ should be met. The ensures us the optimal clusters in each round. Now new probabilities of cluster heads election for different kinds of nodes are made using the residual and average energy of the system. The new probabilities with the concept of DEEC 's residual energy $E_{i}$ and average energy $E_{a}$ (same as of $E(\bar{r}))$ are as: 


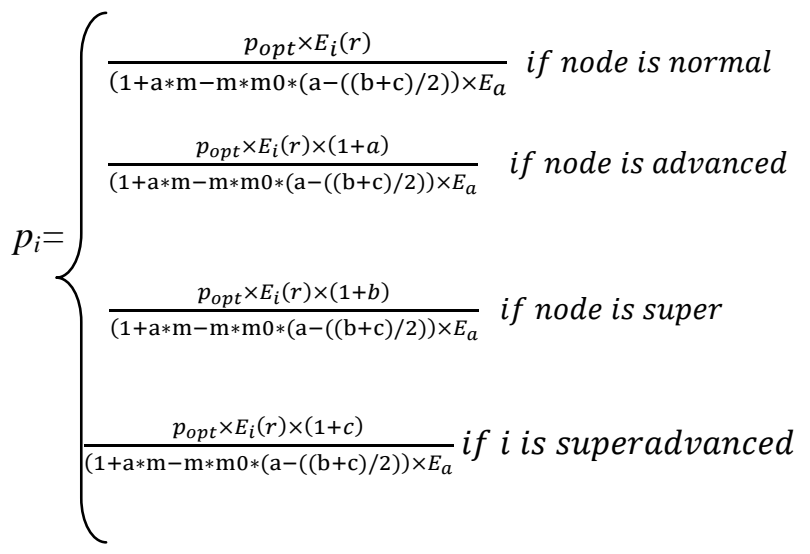

Ultimately our new threshold for deciding the cluster heads election is as:

$$
\begin{aligned}
& T(i)=\left\{\begin{array}{l}
\frac{p_{\text {norm }}}{1-p_{\text {norm }}\left(r \times \bmod \left(\frac{1}{p_{\text {norm }}}\right)\right)} \text { ifi is normal and } i \in G \\
\frac{p_{\text {adv }}}{1-p_{\text {adv }}\left(r \times \bmod \left(\frac{1}{p_{\text {adv }}}\right)\right)} \text { if } i \text { is advanced and } i \in G^{\prime} \\
\frac{p_{\text {sup }}}{1-p_{\text {sup }}\left(r \times \bmod \left(\frac{1}{p_{\text {sup }}}\right)\right)} \quad \text { if } i \text { is super and } i \in G^{\prime \prime} \\
\frac{p_{\text {sadv }}}{1-p_{\text {sadv }}\left(r \times \bmod \left(\frac{1}{p_{\text {sadv }}}\right)\right)} \text { if } i \text { is superadvanced } \\
\text { and } i \in G^{\prime \prime \prime}
\end{array}\right. \\
& 0 \quad \text { otherwise }
\end{aligned}
$$

Now, cluster heads are made according to eq. 3 . We randomly take a number $g$ between $[0,1]$. If the threshold $\mathrm{T}(\mathrm{i})$ for ith node is greater than $\mathrm{g}$ and node $\mathrm{i}$ belongs to set ( $\mathrm{G}$ or $\mathrm{G}^{\prime}$ or G' or G',')then it become cluster head otherwise it will be a simple node. Here G, G', G' and G'" are set of normal, advanced ,super and superadvanced nodes respectively which has not become cluster heads yet. Cluster heads gather the data from its cluster members and they will not send sensed data to Base station as they receive the value. The TEEN is implemented in the nodes. These cluster heads nodes store two threshold hard and soft thresholds. Hard threshold (h) is calculated over highest and lowest value sensed by the nodes. For example in temperature sensing Applications the hard threshold is calculated as the average of maximum temperature sensed and minimum temperature sensed. In our scenario we have simulated our network as temperature sensing wireless sensor network and hard Threshold is taken as 100 (in degree Celsius). Also, we are using the term data for the temperature sensed by the nodes. The sensed value is stored as a variable in the node, called effective sensed value.

(SV). The nodes will next transmit data only when the following conditions are met:

1. The current value of the sensed data $(\mathrm{CV})$ is greater than the hard threshold. $(\mathrm{CV}>\mathrm{h})$ and
2. The current value of the sensed attribute (CV) differs from SV by an amount equal to or greater than the soft threshold (diff $=\mathrm{CV}-\mathrm{SV})$.

Whenever a node transmits data, SV become the current value of the sensed attribute. Here, in this scheme we have taken $\mathrm{s}=2$ (in degree Celsius). These thresholds are making our scheme to work in reactive way as TEEN as Transmission are not periodically as in LEACH, DEEC or EDEEC. The transmission of data is done after receiving the value and applying the thresholds. So data is sent in a non-periodically fashion according to importance of the sensed data. Thus our scheme TADEEC optimized the communication in the networks and makes the communication energy-efficient.

\subsection{Radio Models}

According to the radio energy dissipation model described in [1] and in order to achieve an acceptable Signal-to-Noise Ratio (SNR) in transmitting an B-bit message over a distance $\mathrm{d}$, the energy required is

$$
\begin{aligned}
& E_{\text {trans }}=\begin{array}{c}
B * E_{\text {elec }}+B * E_{f s} * d^{2} \\
B * E_{\text {elec }}+B * E_{m p} * d^{4}
\end{array} \\
& E_{\text {rev }}=B * E_{\text {elec }}
\end{aligned}
$$

Where $E_{\text {elec }}$ is the energy dissipated per bit to run the transmitter( $\left.E_{\text {trans }}\right) . E_{\text {Yov }}$ is energy dissipated in receiving the bits. $E_{f s}$ and $E_{m p}$ depend on the transmitter amplifier model used, do is reference distance and $\mathrm{d}$ is the distance between the sender and the receiver. In this paper, both the free space $\left(\mathrm{d}^{2}\right.$ power loss) and the multi path fading ( $\mathrm{d}^{4}$ power loss) channel models were used in cluster-Base Station Communication, depending on the distance between the transmitter and the receiver.

\subsection{Flow Chart}

The sequence of events taking place in our scheme is shown in the flow chart in fig.1.This flowchart shows events from electing of cluster heads using eq.3 to the intra-cluster communication. The cluster head send the data to the basestation after checking the condition of hard and soft thresholds. If condition is not specified the cluster head drop the packets received from its member node and wait for further packets of data.

\section{SIMULATION AND RESULTS}

This research is simulated in matlab. The positions are calculated on the basis of manual deployment of the nodes so all nodes know positions of all other nodes in the network. The network is taken as an area of $100 * 100$ square meters. The 100 nodes are deployed on the network. The various parameters of the network are listed in table 1.

Some of the important points regarding our Research are:

- Energy of advanced and super nodes are taken in a and $\mathrm{c}$ times reproof normal energy as in EDEEC but we in our research have not introduced new node i.e.superadvanced with more energy than super node of the EDEEC.

We have taken energy of nodes as

normal : normal[EDEEC]

advanced : advanced[EDEEC] 
super : energy between advanced and super nodes of EDEEC

superadvanced : super[EDEEC]

- We have worked on two performance parameters: Allive nodes (or Dead nodes (reverse of alive nodes)) and throughput (packets send to the Base station by cluster heads per round)

- Cluster heads are not sending data on periodical basis (reporting time[1]) but on the basis of hard and soft threshold applied.

In our simulation we have worked on three parameters to make better than previous schemes which are:

- Stability period: the round in which first node get dead. It is very high in TADEEC

- Lifetime: the lifetime of the network. It is the maximum no of rounds in which nodes are alive.

- Throughput: this is no of packets send per round from cluster heads to basestation.

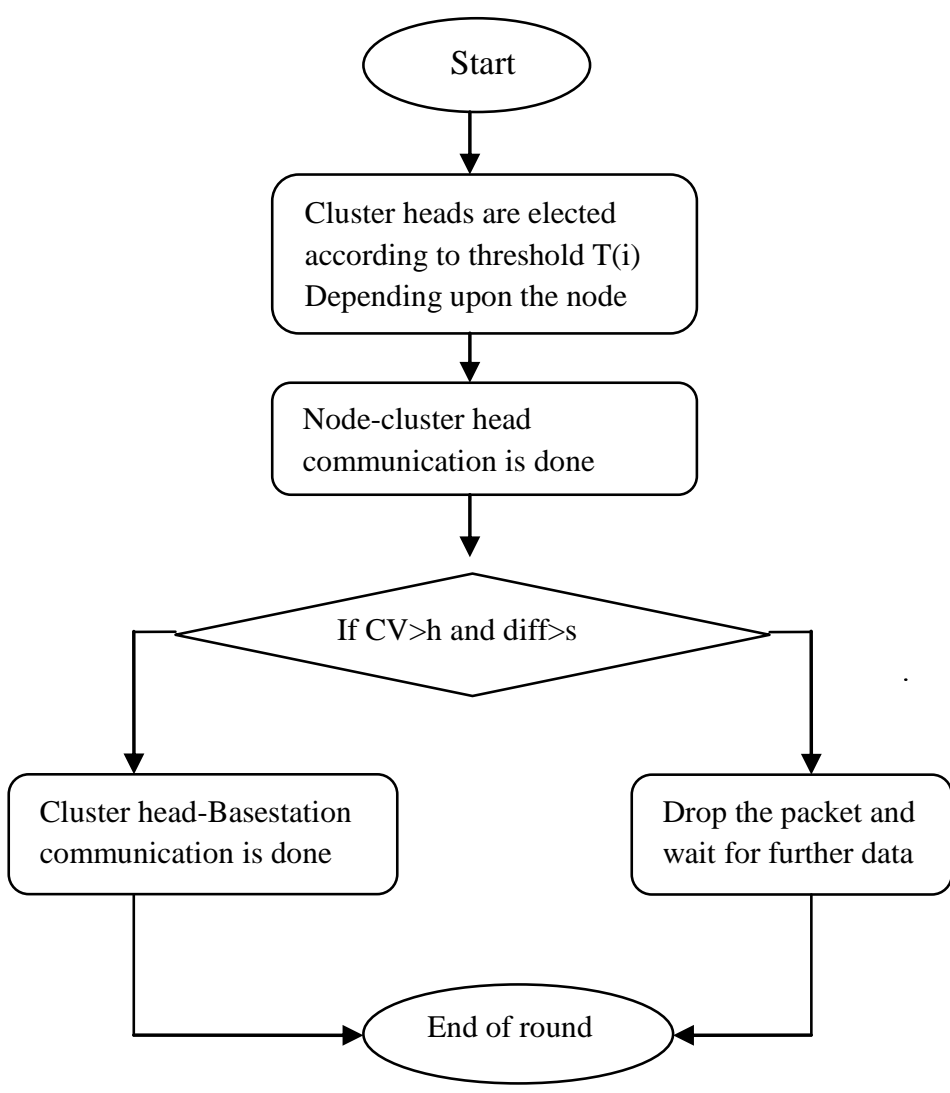

Fig 1: Flowchart showing the steps of routing using proposed protocol

Figures 2 and 3 shows the allive nodes and dead nodes existence respectively in network with increasing rounds in different protocols such as LEACH,DEEC,EDEEC and TADEEC and figure 4 shows the packets send from cluster heads to the basesation. TADEEC is performing better than previous protocols in all these graphs.
Table 1: lists of simulation parameters

\begin{tabular}{|l|l|}
\hline Parameters & Value \\
\hline Area & $100 * 100$ square meters \\
\hline BaseStation & $(50,50)($ in $\mathrm{m})$ \\
\hline Initial Energy & $0.5 \mathrm{~J}$ \\
\hline Transmission Energy & $50 \mathrm{~nJ} / \mathrm{bit}$ \\
\hline Receiver Energy & $50 \mathrm{~nJ} / \mathrm{bit}$ \\
\hline No Of Nodes & 100 \\
\hline Free space Amp Energy & $10 \mathrm{pJ} / \mathrm{bit} / \mathrm{m}^{2}$ \\
\hline Multipath Amplification Energy & $0.0013 \mathrm{pJ} / \mathrm{bit} / \mathrm{m}^{4}$ \\
\hline Message Size(B) & $4000 \mathrm{bits}$ \\
\hline Round & 10000 \\
\hline Aggregation Energy & $5 \mathrm{~nJ} / \mathrm{bit} / \mathrm{packet}$ \\
\hline Hard Threshold & $100 \mathrm{degree}$ Celsius \\
\hline Soft threshold & $2 \mathrm{degree} \mathrm{Celsius}$ \\
\hline Popt & 0.1 \\
\hline M & 0.2 \\
\hline m0 & \\
\hline
\end{tabular}

It has maintained longevity of the nodes in the network to large no of of rounds (even greater than maximum number of rounds we have in our simulations). TADEEC has established a high stability period which can be seen from table 3 . Table 3 has listed the stability period for LEACH,DEEC,EDEEC and TADEEC which is clearly stating the stability period is very high for TADEEC. For TADEEC, it takes even more than double time of DEEC for a node to be dead. Also our third parameter i.e. lifetime is also increased by the implementation of TADEEC in the nodes. Table 4 is showing drastic improvement in lifetime of the network in various simulations of TADEEC which is greater than the LEACH,DEEC and EDEEC. EDEEC definitely worked on to increase lifetime of the network and is better than DEEC but it has been observed in few simulations that stability period for DEEC is more than EDEEC. But TADEEC has outperformed DEEC and EDEEC in all parameters such as stability period, lifetime and throughput. Figure 4 shows the data transmitted to basstataion from cluster heads. The throughput of network for different protocols is stated in table 4. TADEEC has highest data throughput with 27 packets are sent per round in network. 


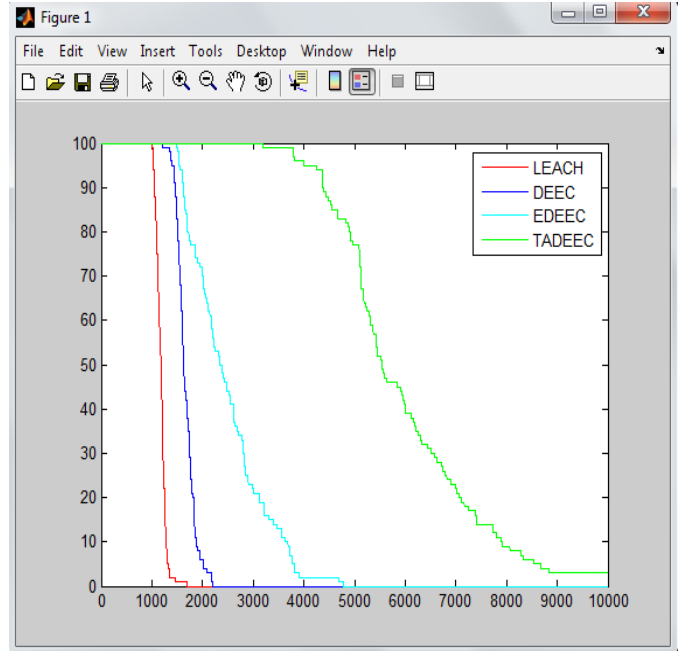

Fig 2: Shows the no of allive nodes vs rounds in the network.

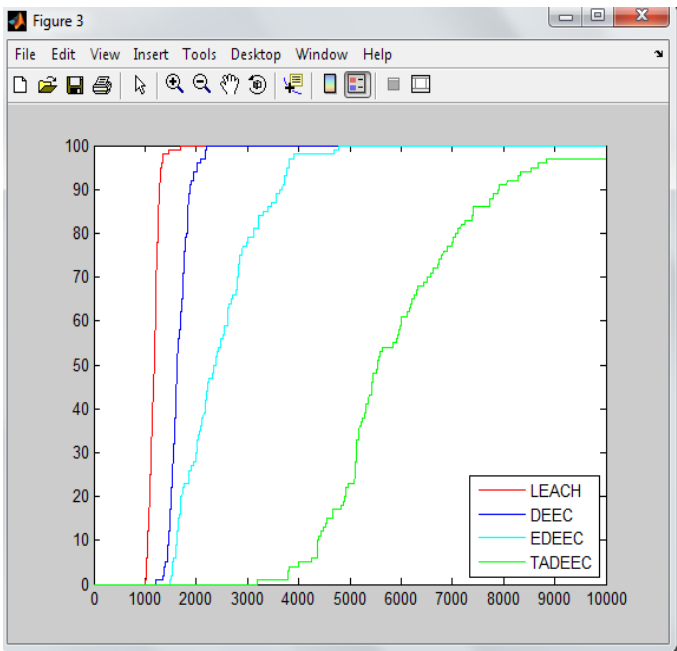

Fig 3: Shows the no of dead nodes vs. rounds

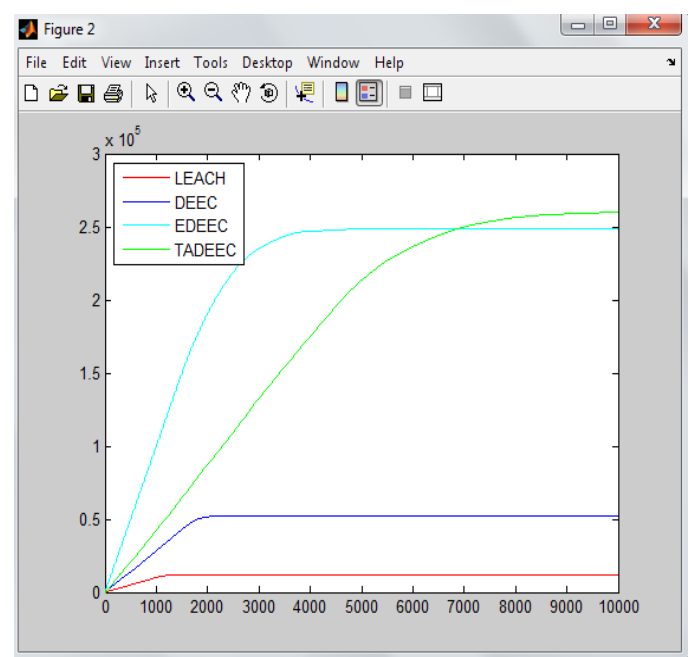

Fig 4: Shows the Packets from cluster heads to Base station
Table 2. Showing the round number in which first node get dead (stability period)

\begin{tabular}{|l|l|l|l|l|}
\hline Protocol & LEACH & DEEC & EDEEC & TADEEC \\
\hline Simulation 1 & 987 & 1266 & 1276 & 3300 \\
\hline Simulation 2 & 983 & 1272 & 1457 & 3586 \\
\hline Simulation 3 & 937 & 1244 & 1304 & 3538 \\
\hline Simulation4 & 990 & 1296 & 1448 & 3600 \\
\hline
\end{tabular}

Table 3. Showing the round number in which last node get dead (lifetime of network)

\begin{tabular}{|l|l|l|l|l|}
\hline Protocol & LEACH & DEEC & EDEEC & TADEEC \\
\hline Simulation 1 & 1400 & 2290 & 4574 & $>10000$ \\
\hline Simulation 2 & 1884 & 2206 & 4838 & 9560 \\
\hline Simulation 3 & 2096 & 2114 & 4761 & $>10000$ \\
\hline Simulation4 & 2266 & 2626 & 4595 & 9954 \\
\hline
\end{tabular}

Table 4. Showing the network throughput for various protocols

\begin{tabular}{|l|l|}
\hline Protocol & Throughput(packets/round) \\
\hline LEACH & 2 \\
\hline DEEC & 5 \\
\hline EDEEC & 25 \\
\hline TADEEC & 27 \\
\hline
\end{tabular}

\section{CONCLUSION}

EDEEC properly used the heterogeneity of nodes for improving the lifetime of the network. We in this paper followed same approach of increasing the heterogeneity of nodes but with the application of an optimization routing protocol. Thus we can see that this optimization algorithm improved the communication among the heterogeneous nodes and prolong the lifetime of the nodes to maximize the communication. Thus this paper is successful in achieving hih stability period, lifetime and throughput of the network.This paper provided a better transmission approach while taking care of limited-energy nodes. The simulation outperformed the LEACH, DEEC and EDEEC and gave a better mechanism for routing in the wireless sensor network.

\section{REFERENCES}

[1] W.Heinzelman, A. Chandrakasan, and H. Balakrishnan, "Energy-efficient communication protocol for wireless sensor networks," in the Proceeding of the Hawaii International Conference System Sciences, Hawaii, January 2000 .

[2] W.R. Heinzelman, A.P. Chandrakasan, H. Balakrishnan, "An applicationspecific protocol architecture for wireless microsensor networks", IEEE Transactions on Wireless Communications 1 (4) (2002) 660-670.

[3] Lindsey S,Raghavenda CS.PEGASIS : Power efficient gathering in sensor informationsystems[C].Williamson DA.Proc of the IEEE Aerospace Conf.New York:IEEE Press, 2002:1125-1130.

[4] G. Smaragdakis, I. Matta, A. Bestavros, "SEP: A Stable Election Protocol for clustered heterogeneous wireless sensor networks", in: Second International Workshop on Sensor and. Actor Network Protocols and Applications (SANPA 2004), 2004 
[5] Aderohunmu, F.A. and Deng, J.D., "An Enhanced Stable Election Protocol (ESEP) for Clustered Heterogeneous WSN".

[6] S. Faisal, N. Javaid, A. Javaid, M. A. Khan, S. H. Bouk and Z. A. Khan, "Z-SEP: Zonal-Stable Election Protocol for Wireless Sensor Networks" Journal of Basic and Applied Scientific Research (JBASR), 2013.

[7] L. Qing, Q. Zhu, M. Wang, "Design of a distributed energy-efficient clustering algorithm for heterogeneous wireless sensor networks". ELSEVIER, Computer Communications 29, 2006, pp 2230- 2237.

[8] Elbhiri, B. , Saadane, R. , El Fkihi, S. , Aboutajdine, D. "Developed Distributed Energy-Efficient Clustering (DDEEC) for heterogeneous wireless sensor networks", in: 5th International Symposium on I/V Communications and Mobile Network (ISVC), 2010.

[9] Parul Saini, Ajay.K.Sharma, "E-DEEC- Enhanced Distributed Energy Efficient Clustering Scheme for heterogeneous WSN", in: $20101^{\text {st }}$ International Conference on Parallel, Distributed and Grid Computing (PDGC - 2010).
[10] T. N. Qureshi, N. Javaid, M. Malik, U. Qasim, Z. A. Khan "On Performance Evaluation of Variants of DEEC in WSNs"In Seventh International Conference on Broadband, Wireless Computing, Communication and Applications 2012

[11] Parul Saini, Ajay.K.Sharma, "Energy Efficient Scheme for Clustering Protocol Prolonging the Lifetime of Heterogeneous Wireless Sensor Networks", International Journal of Computer Applications (0975 8887), Volume 6 No.2, September 2010.

[12]A. Manjeshwar and D. P. Agarwal, "TEEN: a routing protocol for enhanced efficiency in wireless sensor networks," In 1st International Workshop on Parallel and Distributed Computing Issues in Wireless Networks and Mobile Computing, April 2001.

[13] T. J. Shepard. A channel access scheme for large dense packet radio networks. In Proccedings of ACM SIGCOMM, pages 219230, September 1996.

[14] MATLAB 7.4.0(R2007a) www.mathworks.com 